\title{
Development and validation of a sunlight exposure questionnaire for urban adult Filipinos
}

\author{
Marc Gregory Yu' ${ }^{1}$ Nina Castillo-Carandang ${ }^{2,3}$, Maria Elinor Grace Sison ${ }^{4}$, Angelique Bea Uy ${ }^{5}$, \\ Katrina Lenora Villarante ${ }^{6}$, Patricia Maningat' ${ }^{1}$, Elizabeth Paz-Pacheco ${ }^{1}$, Eileen Abesamis-Cubillan ${ }^{4}$ \\ 'Section of Endocrinology, Diabetes and Metabolism, Department of Medicine, Philippine General Hospital, University of the Philippines, \\ Manila, Philippines; ${ }^{2}$ Department of Clinical Epidemiology, University of the Philippines College of Medicine, Manila, Philippines; ${ }^{3}$ Institute of \\ Clinical Epidemiology, University of the Philippines-National Institutes of Health, Manila, Philippines; ${ }^{4}$ Section of Dermatology, Department of \\ Medicine, Philippine General Hospital, University of the Philippines, Manila, Philippines; ${ }^{5}$ Department of Medicine, Philippine General Hospital, \\ University of the Philippines, Manila, Philippines; ${ }^{6}$ Department of Family and Community Medicine, Philippine General Hospital, University of \\ the Philippines, Manila, Philippines
}

OBJECTIVES: To develop and validate a self-reported sunlight exposure questionnaire (SEQ) for urban adult Filipinos.

METHODS: The study included adults (19-76 years old) in Metro Manila, Philippines, well-versed in the Filipino (Tagalog) language and had resided in Metro Manila for at least 1 year. Exclusion criteria included pregnancy, active skin disorders, and immunocompromised states. An expert panel created a questionnaire in Likert-scale format based on a conceptual framework and 4 existing instruments. The study proceeded in 4 phases: questionnaire item development, translation and back-translation, pretesting, and construct validity and reliability testing using factor analysis, the Cronbach alpha coefficient, and the paired t-test.

RESULTS: A 25-item, self-administered, Filipino (Tagalog) SEQ answerable using a 4-point Likert scale was created. The questionnaire was administered to 260 adult participants twice at a 2-week interval, with all participants completing both the first and second rounds of testing. All questionnaire items possessed adequate content validity indices of at least 0.86 . After factor analysis, 3 questionnaire domains were identified: intensity of sunlight exposure, factors affecting sunlight exposure, and sun protection practices. Internal consistency was satisfactory for both the overall questionnaire (Cronbach alpha, 0.80) and for each of the domains (Cronbach alpha, $0.74,0.71$, and 0.72 , respectively). No statistically significant differences were observed in the responses between the first and second rounds of testing, indicating good test-retest reliability.

CONCLUSIONS: We developed a culturally-appropriate SEQ with sufficient content validity, construct validity, and reliability to assess sunlight exposure among urban adult Filipinos in Metro Manila, Philippines.

KEY WORDS: Vitamin D deficiency, Sunlight, Surveys and questionnaires, Philippines

\footnotetext{
Correspondence: Marc Gregory Yu

Section of Endocrinology, Diabetes and Metabolism, Department of Medicine, Philippine General Hospital, University of the Philippines, Taft Avenue, Ermita, Manila 1000, Philippines E-mail: marcgreggy@yahoo.com

Received: Jul 25, 2018 / Accepted: Oct 11, 2018 / Published: Oct 11, 2018

This article is available from: http://e-epih.org/

(C) This is an open-access article distributed under the terms of the Creative Commons Attribution License (http://creativecommons.org/licenses/by/4.0/), which permits unrestricted use, distribution, and reproduction in any medium, provided the original work is properly cited.

(C) 2018, Korean Society of Epidemiology
}

\section{INTRODUCTION}

Vitamin D deficiency (VDD) is a major public health concern [1]. It is reflected by low serum 25-hydroxyvitamin D (25-OHD) levels, which lead to adverse changes in calcium and phosphate homeostasis and increased fracture risk. In the Philippines, a 2009 study of postmenopausal women found that $36 \%$ of the participants had insufficient $25-\mathrm{OHD}$ levels (20-30 ng/mL), but only $30 \%$ of those women received calcium and vitamin D supplementation [2]. Another study of 369 randomly selected Metro Manila office workers in 2014 revealed that $58 \%$ of the participants had deficient serum $25-\mathrm{OHD}$ levels $(<20 \mathrm{ng} / \mathrm{mL})$, while $30 \%$ had insuffcient levels [3]. 
Exposure to ultraviolet rays (UVB) is the main source of vitamin $\mathrm{D}$ in humans. This is because the enteral route is not a good source of vitamin D unless foods are fortified with vitamin D [4]. A contributing factor to the increasing prevalence of VDD in the Philippines is rapid urbanization, which has resulted in more young adults having indoor jobs and thus less sun exposure, raising concerns about bone health during the period when they are achieving peak bone mass. Furthermore, air pollution in major Philippine cities decreases the amount of UVB that reaches the earth's surface [5].

A major limitation in the area of VDD research is the lack of an appropriate, inexpensive, and easily-administered tool for measuring sunlight exposure [6]. Compared with other methods, questionnaires are considered to be the most cost-effective way of measuring sunlight exposure in population-based studies [7]. Of the available sunlight exposure questionnaires (SEQs), only 2 were validated in Asia (Hong Kong and Pakistan) [6,7] and only 3 were created in the context of VDD by correlating the questionnaire results with serum 25-OHD levels, showing moderate correlations $[6,8,9]$. At present, there is no existing SEQ that has been validated for Southeast Asian or tropical populations. This study aimed to develop and validate a culturally-appropriate, self-reported SEQ for urban adult Filipinos.

\section{MATERIALS AND METHODS}

\section{Study participants}

The study included individuals $>19$ years old who were fluent in the Filipino (Tagalog) language, lived in Metro Manila at least 5 days a week for at least 1 year, and provided written informed consent. We set 1 year as the minimum duration of urban living to account for all possible weather changes and seasonal variations. Those who were pregnant or who had known active skin disorders or immunocompromised states potentially affecting sunlight exposure were excluded. Study participants were selected by purposive sampling from any of the 17 component cities of Metro Manila using a sampling frame (Table 1). Based on an existing study on the prevalence of VDD in the Philippines (58\% among 369 participants), with confidence limits of $5 \%$ and a design effect

Table 1. Sampling frame for the study

\begin{tabular}{lcccc}
\hline & \multicolumn{3}{c}{ Working age $(19-60 \mathrm{yr})$} & Elderly/ \\
\cline { 2 - 4 } & $\begin{array}{c}\text { Age } \\
(\mathrm{yr})\end{array}$ & $\begin{array}{c}\text { Professional, } \\
\text { mostly indoor } \\
\text { work }\end{array}$ & $\begin{array}{c}\text { Some educa- } \\
\text { tion, mostly } \\
\text { outdoor work }\end{array}$ & $\begin{array}{c}\text { retirees } \\
(>60 \mathrm{yr})\end{array}$ \\
\hline \multirow{6}{*}{ Male } & $19-30$ & 15 & 15 & 10 \\
& $31-40$ & 15 & 15 & \\
Female & $41-50$ & 15 & 15 & \\
& $51-60$ & 15 & 15 & 10 \\
& $19-30$ & 15 & 15 & \\
& $31-40$ & 15 & 15 & \\
& $41-50$ & 15 & 15 & \\
& $51-60$ & 15 & 15 &
\end{tabular}

of 1.0, an initial sample size of 187 was calculated [3]. However, after development of the final questionnaire, the sample size was increased to 250 since we took into account the recommended 10:1 subject-to-item ratio for factor analysis [10]. Ten additional subjects were recruited to ensure that participants more equally distributed across the various brackets- 15 each for the workingage groups and 10 each for the elderly groups - yielding a final sample size of 260. Epi Info version 7.0 (https://epi-info.software. informer.com/7.0/) was used for sample size calculation.

\section{Study procedures}

The study proceeded in 4 phases.

\section{Phase I: questionnaire item development}

An extensive literature review of concepts on sunlight exposure assessment was performed and relevant questionnaires were identified, using the main keywords "sunlight," "questionnaire," "urban," "vitamin D," and "osteoporosis." There were no restrictions on language, country, or year of publication. Based on their relevance (mainly the inclusion of different sun exposure variables and/or correlation with serum 25-OHD), the SEQs developed by Cargill et al. [9] in Australia, Hanwell et al. [8] in Italy, Humayun et al. [6] in Pakistan, and Wu et al. [7] in Hong Kong were used as references for this study. In addition, an existing conceptual framework on the attitudes, behaviors, and beliefs of urban adult Filipinos on sunlight exposure was also used (Figure 1) [11].

A panel of 3 endocrinologists, 2 dermatologists, a health social scientist, an internist, and a community medicine physician created preliminary questionnaire items using the following guidelines: (1) Is the item unbiased? (2) Is there a strong likelihood that most respondents will answer the item truthfully? (3) Do most respondents possess sufficient knowledge needed to answer the item? (4) Will most respondents be willing to answer the item? (5) Does the item avoid leading respondents to a specific answer? and (6) Is the language used clear and simple enough so that respondents are able to understand all questions? [12].

The questionnaire items were constructed in the form of a 4-point Likert scale and arranged using the following guidelines: (1) Nonsensitive questions were placed at the beginning, since they were assumed to be non-threatening and tended to put the respondent at ease; (2) Items of major interest to the study were also prioritized, since there was greater probability of the respondent completing the first part of the questionnaire; (3) Sensitive items were placed last so that any potential emotions provoked would not influence the responses to other questions; and (4) As much as possible, items on similar topics were placed close to each other [12].

All questionnaire items then underwent content validity assessment by each panel member using a 4-point ordinal scale: 4 , very relevant; 3 , somewhat relevant; 2 , hardly relevant; and 1 , not at all relevant. The content validity index (CVI) of each item was computed as the number of evaluators giving a 3 or 4 divided by the total number of evaluators. Only items with a CVI of at least 0.86 were retained in the questionnaire [13]. 


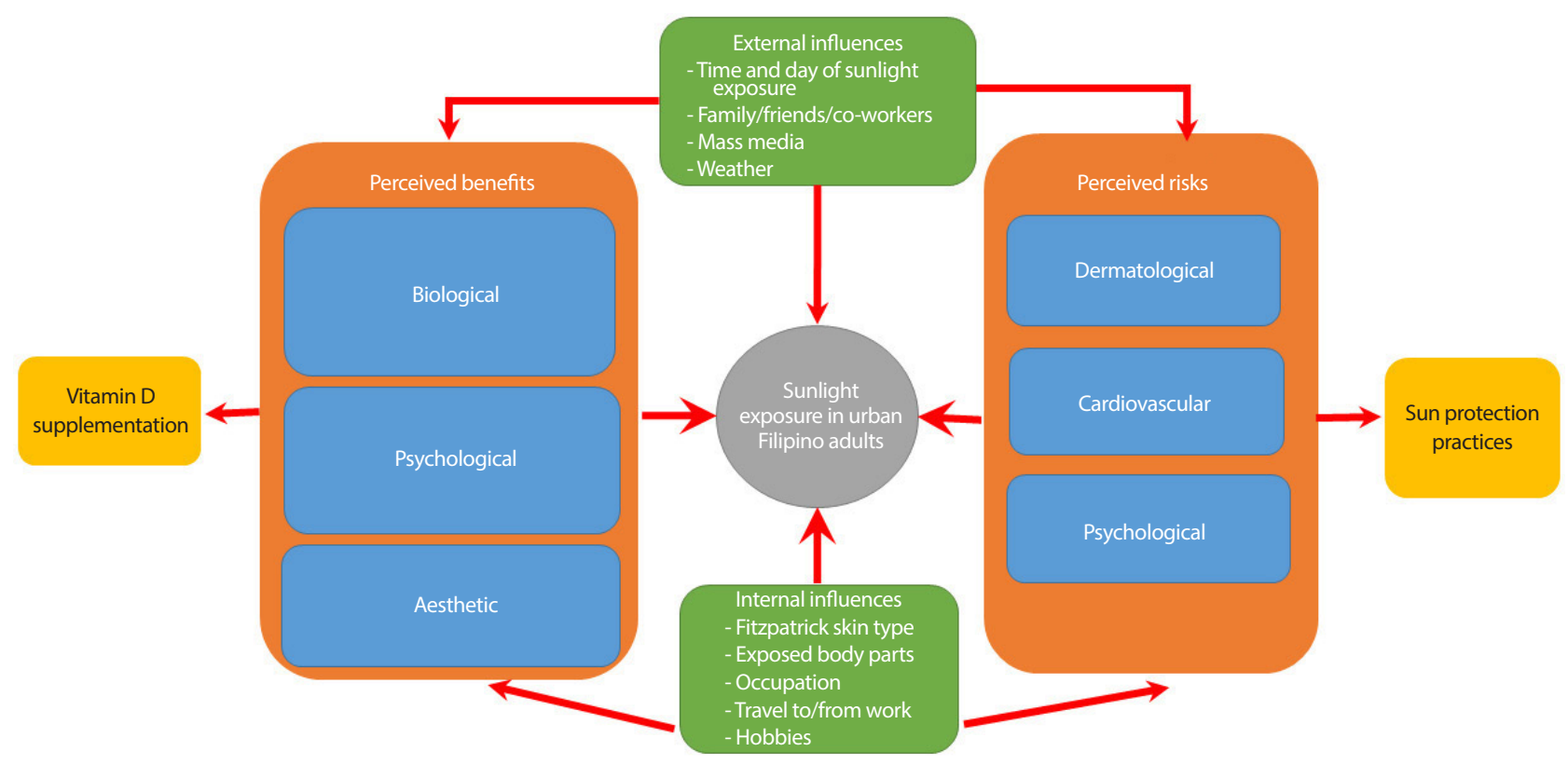

Figure 1. Conceptual framework on the attitudes, behaviors, and beliefs of urban adult Filipinos on sunlight exposure. Adapted from Yu et al. JAFES 2018;33:37-43 [11], on the basis of Open Access.

\section{Phase II: forward-translation and back-translation}

The items of the draft questionnaire were then translated from English to Filipino (Tagalog) by 2 independent bilingual translators, one of whom was a physician with knowledge of the study and its concepts, and the other a non-medical professional with no knowledge of the study or its concepts. The linguistic and cultural quality of the 2 translations was reviewed individually by the panel experts and was consolidated into a single version by consensus. The newly-synthesized Filipino SEQ then underwent backward translation by a different bilingual translator who was uninvolved in the study. Finally, both translated and back-translated versions were reviewed by the panel experts to reach a consensus for the prefinal Filipino (Tagalog) questionnaire version [14].

\section{Phase III: pretest}

Pretesting of the prefinal questionnaire to test flow and comprehensibility was performed by administering it to a sample equivalent to thrice the number of questionnaire items. Questionnaires were self-administered and the time needed to complete the test was noted for each person. After answering the questionnaire, the participants were asked for feedback regarding comprehensibility and format using a cognitive debriefing form. The form utilized both close- and open-ended questions. Revision of the questionnaire was then carried out by the panel members based on pretest results and feedback to create the final Filipino version of the SEQ.

\section{Phase IV: construct validity test and reliability test}

The final questionnaire was administered to the sample population twice at a 2 -week interval, with a similar procedure as that of the pretest. A research assistant was accordingly trained in the
Table 2. Distribution of respondents according to socio-demographic characteristics $(n=260)$

\begin{tabular}{lc}
\hline Characteristics & $\mathrm{n}(\%)$ \\
\hline Age (mean \pm SD, yr) & $41.54 \pm 13.60$ \\
Sex & \\
Female & $130(50.0)$ \\
Male & $130(50.0)$ \\
Duration of urban living in months (mean \pm SD) & $28.77 \pm 18.60$ \\
Educational attainment & \\
Elementary graduate or less & $7(2.7)$ \\
High school graduate or less & $63(24.2)$ \\
Some college/vocational course & $63(24.2)$ \\
College graduate & $127(48.8)$ \\
Type of work & \\
Day shift & $207(79.6)$ \\
Night shift & $34(13.1)$ \\
Unemployed & $19(7.3)$ \\
Location of work & \\
Indoor & $163(62.7)$ \\
Outdoor & $97(37.3)$ \\
Household income (PHP/mo) ${ }^{1}$ & \\
$\geq 10,000^{1}$ & $176(67.7)$ \\
$<10,000^{1}$ & $84(32.3)$ \\
Time needed to complete test in minute (mean $\pm S D)$ & \\
1st test & $6.78 \pm 5.40$ \\
2nd test & $6.61 \pm 2.40$ \\
\hline
\end{tabular}

SD, standard deviation; PHP, Philippine peso.

${ }^{1}$ PHP=US\$ 0.019 . 
recruitment of participants and questionnaire administration. Data from the first test underwent factor analysis to determine the different questionnaire domains; items that possessed similar factor loading values were grouped under a particular domain.

Reliability of the questionnaire was assessed by internal consistency and by test-retest reliability. Internal consistency was analyzed using the Cronbach alpha coefficient, with 0.7 as an acceptable cutoff value [15], while test-retest reliability was analyzed using the paired t-test. Statistical analyses were performed using Stata SE version 13 (StataCorp., College Station, TX, USA). Descriptive statistics included the mean and standard deviation for normally distributed quantitative variables, median and interquartile range for non-normally distributed quantitative variables, and frequency and percentage for qualitative variables. The threshold for statistical significance was set at $5 \%$.

\section{Ethics statements}

Both the study protocol and informed consent forms were approved by the University of the Philippines institutional review board prior to commencement (UPMREB code: MED-2016-00301). Study participants also received a token honorarium for their participation.

\section{RESULTS}

Table 2 summarizes the socio-demographic characteristics of the entire study population.

\section{Phase l: questionnaire item development}

The expert panel devised an initial list of 32 questions. After content validity assessment, only 25 questions were retained since

Table 3. CVI values for items in the Filipino SEQ

\begin{tabular}{|c|c|c|}
\hline No. & Question & CVI \\
\hline 1 & How do you describe your skin when it is exposed to the sun? & 1.00 \\
\hline 2 & What part of your body is usually exposed to the sun? & 1.00 \\
\hline 3 & How long do you usually spend under the sun on a weekday? & 1.00 \\
\hline 4 & How long do you usually spend under the sun on a weekend? & 1.00 \\
\hline 5 & How long do you usually spend under the sun during sunny weather? & 1.00 \\
\hline 6 & How long do you usually spend under the sun during cloudy weather? & 1.00 \\
\hline 7 & How long do you usually spend under the sun during rainy weather? & $0.67^{1}$ \\
\hline 8 & What time of the day are you usually exposed to the sun? & 1.00 \\
\hline 9 & How often do you go out in the sun due to work or daily routine? & 1.00 \\
\hline 10 & How often do you walk or use public transport to do the above activities? & 1.00 \\
\hline 11 & How often do you engage in outdoor activities such as jogging, cycling, and swimming? & 1.00 \\
\hline 12 & How often do you take calcium with vitamin D or multivitamins? & 1.00 \\
\hline 13 & How likely are you to be exposed to the sun to get stronger bones and better health? & 1.00 \\
\hline 14 & How likely are you to be exposed to the sun to get happier and livelier? & 1.00 \\
\hline 15 & How likely are you to be exposed to the sun to get more beautiful skin? & 1.00 \\
\hline 16 & How likely are you to avoid sun exposure due to the influence of family, friends, and coworkers? & 1.00 \\
\hline 17 & How likely are you to avoid sun exposure due to the influence of TV, radio, and internet? & 1.00 \\
\hline 18 & How likely are you to avoid sun exposure due to sunburn, skin cancer, skin allergy, and rashes? & 1.00 \\
\hline 19 & How likely are you to avoid sun exposure due to heat stroke, hypertension, and dizziness? & 1.00 \\
\hline 20 & How likely are you to avoid sun exposure due to sweating and fear of darker skin? & 1.00 \\
\hline 21 & When going out in the sun, how often do you wear long sleeves? & $0.33^{1}$ \\
\hline 22 & When going out in the sun, how often do you wear long pants? & $0.33^{1}$ \\
\hline 23 & When going out in the sun, how often do you wear a hat? & 1.00 \\
\hline 24 & When going out in the sun, how often do you wear sunglasses? & $0.17^{1}$ \\
\hline 25 & When going out in the sun, how often do you use an umbrella? & 1.00 \\
\hline 26 & When going out in the sun, how often do you walk under the shade? & 1.00 \\
\hline 27 & When going out in the sun, how often do you use transportation with closed windows? & $0.17^{1}$ \\
\hline 28 & When going out in the sun, how often do you use sunscreen containing at least SPF $30 ?$ & 1.00 \\
\hline 29 & When do you usually apply sunscreen? & 1.00 \\
\hline 30 & How much sunscreen do you usually apply? & 1.00 \\
\hline 31 & How often do you use a sunlamp? & $0.00^{1}$ \\
\hline 32 & How often do you use a sunbed? & $0.00^{1}$ \\
\hline
\end{tabular}

$\mathrm{CVI}$, content validity index; SEQ, sunlight exposure questionnaire; SPF, sun protection factor.

'Eventually omitted from the questionnaire due to $\mathrm{CVI}<0.86$. 
they had CVIs of at least 0.86 (Table 3). Question \#7 was removed because the panel experts agreed that sunlight exposure during rainy weather is minimal; questions \#21 and \#22 were considered redundant since question $\# 2$ already inquired regarding clothing and body part exposure; question \#24 was removed since wearing sunglasses was considered more for protection from the sun's glare; question \#27 was also considered redundant since question \#10 already inquired about the respondent's usual mode of transport; and questions \#31 and \#32 were removed since the use of sunlamps and sunbeds is not common among Filipinos.

\section{Phase Il: forward-translation and back-translation}

During the translation process, words like "jogging," "calcium," "vitamin D," "multivitamins," "sunburn," "allergy," "heat stroke," and "sunscreen" were retained in English, as these were deemed familiar terms for ordinary Filipinos. No significant disparities between the English and Filipino (Tagalog) versions were detected by the independent bilingual translators during translation and back-translation.

\section{Phase III: results of pretest}

Pretesting of the prefinal Filipino (Tagalog) SEQ was conducted with 75 participants. The average time needed to complete the 25item questionnaire was 15 minutes (range, 5-30 minutes). In general, the respondents found the questionnaire to be comprehensible with acceptable length and arrangement. No questions were considered sensitive, biased, or threatening during cognitive debriefing. For questions \#17-\#19, the phrase "posibilidad ng" ("possibility of") was added to the beginning of each question to emphasize the risk of sunlight exposure. For questions \#23-\#25, many respondents preferred the term "sunblock" to "sunscreen." However, "sunscreen" was retained, as it affords protection from the entire ultraviolet range, as opposed to "sunblock," which only affords protection from UVB. Furthermore, several guidelines, including those of the US Food and Drug Administration, do not recommend the word "sunblock," as it may falsely overemphasize a product's efficacy [16].

\section{Phase IV: results of construct validity test and reliability test}

The final questionnaire was administered to the entire sample of 260 participants. There were no dropouts; all participants completed both the first and second rounds of testing. The mean age was 41.54 years, with a mean duration of urban living of 28.77

Table 4. Factor loadings by factor analysis of items of the Filipino SEQ

\begin{tabular}{|c|c|c|c|c|}
\hline \multirow{2}{*}{ Item no. } & & \multicolumn{3}{|c|}{ Factor } \\
\hline & & 1 & 2 & 3 \\
\hline 1 & How do you describe your skin when it is exposed to the sun? & -0.25 & 0.10 & -0.06 \\
\hline 2 & What part of your body is usually exposed to the sun? & 0.20 & 0.20 & 0.01 \\
\hline 3 & How long do you usually spend under the sun on a weekday? & 0.27 & 0.57 & -0.16 \\
\hline 4 & How long do you usually spend under the sun on a weekend? & 0.22 & 0.59 & -0.15 \\
\hline 5 & How long do you usually spend under the sun during sunny weather? & 0.31 & 0.47 & -0.15 \\
\hline 6 & How long do you usually spend under the sun during cloudy weather? & 0.24 & 0.26 & -0.12 \\
\hline 7 & What time of the day are you usually exposed to the sun? & 0.28 & 0.31 & 0.17 \\
\hline 8 & How often do you go out in the sun due to work or daily routine? & 0.43 & 0.16 & -0.24 \\
\hline 9 & How often do you walk or use public transport to do the above activities? & 0.19 & 0.17 & 0.12 \\
\hline 10 & How often do you engage in outdoor activities such as jogging, cycling, and swimming? & 0.18 & 0.13 & -0.12 \\
\hline 11 & How often do you take calcium with vitamin D or multivitamins? & 0.05 & -0.03 & -0.23 \\
\hline 12 & How likely are you to be exposed to the sun to get stronger bones and better health? & 0.62 & 0.10 & -0.61 \\
\hline 13 & How likely are you to be exposed to the sun to get happier and livelier? & 0.59 & -0.11 & -0.59 \\
\hline 14 & How likely are you to be exposed to the sun to get more beautiful skin? & 0.43 & -0.13 & -0.48 \\
\hline 15 & How likely are you to avoid sun exposure due to the influence of family, friends, and coworkers? & 0.23 & 0.10 & 0.17 \\
\hline 16 & How likely are you to avoid sun exposure due to the influence of TV, radio, and internet? & 0.19 & 0.14 & 0.17 \\
\hline 17 & How likely are you to avoid sun exposure due to sunburn, skin cancer, skin allergy, and rashes? & 0.64 & -0.57 & 0.18 \\
\hline 18 & How likely are you to avoid sun exposure due to heat stroke, hypertension, and dizziness? & 0.61 & -0.53 & 0.05 \\
\hline 19 & How likely are you to avoid sun exposure due to sweating and fear of darker skin? & 0.46 & -0.09 & 0.13 \\
\hline 20 & When going out in the sun, how often do you wear a hat? & -0.13 & -0.05 & 0.26 \\
\hline 21 & When going out in the sun, how often do you use an umbrella? & 0.22 & 0.10 & 0.43 \\
\hline 22 & When going out in the sun, how often do you walk under the shade? & 0.24 & 0.03 & 0.33 \\
\hline 23 & When going out in the sun, how often do you use sunscreen containing at least SPF $30 ?$ & 0.35 & 0.26 & 0.65 \\
\hline 24 & When do you usually apply sunscreen? & 0.44 & 0.27 & 0.63 \\
\hline 25 & How much sunscreen do you usually apply? & 0.39 & 0.31 & 0.65 \\
\hline
\end{tabular}

$\mathrm{SEQ}$, sunlight exposure questionnaire; SPF, sun protection factor. 
Table 5. Per-domain test-retest reliability of the Filipino SEQ

\begin{tabular}{lccc}
\hline Sunlight exposure domains & First test & Retest & p-value \\
\hline Overall for questionnaire & $2.39 \pm 0.36$ & $2.40 \pm 0.37$ & 0.73 \\
$\begin{array}{c}\text { Domain 1 (intensity of sunlight } \\
\text { exposure) }\end{array}$ & $1.92 \pm 0.49$ & $1.90 \pm 0.45$ & 0.54 \\
$\begin{array}{c}\text { Domain 2 (factors affecting } \\
\text { sunlight exposure) }\end{array}$ & $2.47 \pm 0.43$ & $2.50 \pm 0.45$ & 0.34 \\
$\begin{array}{c}\text { Domain 3 (sun protection } \\
\text { methods) }\end{array}$ & $2.77 \pm 0.65$ & $2.76 \pm 0.64$ & 0.96 \\
\hline
\end{tabular}

Values are presented as mean \pm standard deviation.

SEQ, sunlight exposure questionnaire.

months. The majority of the respondents were day shift (79.6\%) and indoor $(62.7 \%)$ workers. There was no significant difference in the mean time needed to complete the first (6.78 minutes) and the second (6.61 minutes) test (Table 2).

Factor analysis yielded 3 principal component factors corresponding to the different questionnaire domains. Items that possessed similar factor loading values were grouped under a particular domain. The 3 domains were labeled: (1) intensity of sunlight exposure (containing questions \#1-\#7); (2) factors affecting sunlight exposure (containing questions \#8-\#19); and (3) sun protection practices (containing questions \#20-\#25). Table 4 shows the 3 domains of the SEQ and the factor loading values of the items in each domain.

The internal consistency assessment yielded an overall Cronbach alpha coefficient of 0.80 , indicating that the questionnaire generally showed internal consistency. The 3 domains were internally consistent on their own as well, with coefficient values of $0.74,0.71$, and 0.72 , respectively. Similarly, the paired t-test yielded no statistically significant differences between the responses obtained in the first and second rounds of testing for either the entire questionnaire or each of its domains, indicating satisfactory testretest reliability (Table 5).

\section{DISCUSSION}

This is the first SEQ developed and validated for use in an urban adult Filipino population. The questionnaire was designed to assess the intensity of sunlight exposure, the various factors affecting sunlight exposure, and the different sunlight protection practices utilized by urban adult Filipinos.

To ensure adequate representativeness of the sample, our sampling frame took into account age, sex, educational attainment, work shift and location, and economic status. Although it could be argued that elderly respondents should comprise a greater proportion of the sample (given that the consequences of VDD are especially strongly felt in this population), our aim was to create a more even distribution of respondents across the entire adult lifespan to maximize the questionnaire's applicability [17]. The respondents' locations within Metro Manila were not part of the sampling frame, since each of the Philippine capital's 17 component cities are topographically similar, and hence there were no expected sig- nificant differences in sunlight exposure. The Köppen climate classification lists Metro Manila as having a uniformly tropical wet and dry climate [18].

The questionnaire development process drew on the existing instruments of Cargill et al. [9] in Australia, Hanwell et al. [8] in Italy, Humayun et al. [6] in Pakistan, and Wu et al. [7] in Hong Kong. Although the questionnaires served as important references, no questions were directly taken from any of these instruments, as they were all developed in countries of a different ethnicity, geography, and climate compared to the Philippines. Hence, we utilized a separate conceptual framework that explored additional aspects of sunlight exposure in Filipinos that may not have been covered in the existing questionnaires [11]. A unique feature of our questionnaire is the inclusion of questions pertaining to the perceived risks and benefits of sunlight exposure, which are significant determinants of an individual's sunlight exposure practices. We also added questions pertaining to the influences of other people and mass media on sunlight exposure, given the strong kinship and social ties among Filipinos and the widespread use of technology by urban residents [19]. A current disadvantage of the questionnaire is the lack of a validated scoring system and the lack of correlation with established gold standard measurements, the latter of which will be addressed in the next phase of the study.

In the construction of questionnaire items, we utilized the Likert scale, the most widely-used approach to scaling responses in questionnaire research. Unlike simple close-ended questions, the Likert scale has the ability to specify levels of agreement or disagreement in a symmetric fashion, capturing the range of intensity of feelings for a given item, which is then simplified as the sum of the questionnaire items [20]. Questionnaires in Likert scale format are also easy to use and allow more variables in a study because the format enables respondents to answer more questions in the same time required to answer fewer open-ended questions $[21,22]$. While we retained the same choices for many questions ("never," "rarely," "often," and "always"), the choices for other questions were crafted to reflect a similarly symmetric degree of sun exposure. This was especially true for questions involving the Fitzpatrick skin classification, body part exposure, and temporal exposure.

During the translation and back-translation process, the independent bilingual translators decided to retain several words in English. This is due to the fact that these particular words are considered familiar terms for ordinary Filipinos. In the 2010 Test of English as a Foreign Language, the Philippines ranked 35th out of 163 countries worldwide, and ranked second-best in Asia after Singapore [23].

The questionnaire was assessed using content validity, construct validity, and reliability. Content validity, which refers to the representativeness or relevance of the questionnaire content, was assessed individually by the members of an expert panel [7]. These members were chosen from diverse disciplines to ensure a holistic clinical and psychosocial evaluation of the questionnaire items. While majority of the items possessed sufficient content validity, those that were removed were mostly either redundant or found 
to be non-contributory to sunlight exposure evaluation. Others (such as the use of sunlamps or sunbeds) were deemed not applicable to Filipino culture.

Our questionnaire also possessed satisfactory construct validity. The 3 domains extracted after factor analysis corresponded well with the themes identified during creation of the conceptual framework. Specifically, the first 2 domains corresponded to the influences and perceived benefits and risks of sunlight exposure. The third domain also corresponded to perceived risks, as an increased awareness of these risks leads to an increased usage of sun protection practices (Figure 1). Furthermore, the factor analysis results also fulfilled the rule of having a minimum of 5 questions per domain to enable psychometric testing, with 7 questions in the first domain, 12 questions in the second domain, and 6 questions in the third domain [24]. Reliability, meanwhile, was likewise sufficient in terms of both internal consistency and test-retest reliability. For the latter, the decision to administer the questionnaire 2 weeks apart was made because that time frame was long enough for the participants to not remember their responses from the first test, while being short enough to not allow significant physiological changes to occur. There was also no significant difference in the time needed to complete the first and second rounds of testing, attesting to the questionnaire's consistency in ease of administration.

This study serves as part of a larger project that will eventually involve concurrent and criterion validity assessment of the questionnaire results with established objective parameters, such as dosimetry and serum 25-OHD levels. We also recommend future studies investigating the applicability of the questionnaire to a wider population, particularly rural and other urban areas in the Philippines, in addition to other Southeast Asian and tropical countries of similar ethnicity and geographical latitude.

In conclusion, this study showed that a linguistically and culturally appropriate SEQ possessed sufficient content validity, construct validity, and reliability to assess sunlight exposure among urban adult Filipinos in Metro Manila. The questionnaire results can be eventually applied to evaluate associations with serum 25 OHD levels.

\section{CONFLICT OF INTEREST}

The authors have no conflicts of interest to declare for this study.

\section{ORCID}

Marc Gregory Yu: http://orcid.org/0000-0002-0376-9574; Nina Castillo-Carandang: $h t t p: / / o r c i d . o r g / 0000-0001-5360-1801$; Maria Elinor Grace Sison: http://orcid.org/0000-0002-1222-8817; Angelique Bea Uy: http://orcid.org/0000-0002-9539-8239; Katrina Lenora Villarante: $h t t p: / / o r c i d . o r g / 0000-0001-8691-2403$; Patricia Maningat: http://orcid.org/0000-0003-1984-9277; Elizabeth Paz-Pacheco: http://orcid.org/0000-0003-0232-890X; Eileen Abesamis-Cubillan: http://orcid.org/0000-0001-5417-8013

\section{REFERENCES}

1. Holick MF. The role of vitamin D for bone health and fracture prevention. Curr Osteoporos Rep 2006;4:96-102.

2. Raso AA, Navarra SV, Li-Yu J, Torralba TP. Survey of vitamin D levels among post-menopausal Filipino women with osteoporosis. Int J Rheum Dis 2009;12:225-229.

3. Orosa RL. 3 out of 5 Filipinos suffer from Vit-D deficiency; 2014 [cited 2018 Nov 5]. Available from: http://www.philstar.com/ health-and-family/2014/05/13/1322297/3-out-5-filipinos-suffer-vit-d-deficiency.

4. Beadle PC. Sunlight, ozone, and vitamin D. Br J Dermatol 1977; 97:585-591.

5. Nimitphong H, Holick MF. Vitamin D status and sun exposure in Southeast Asia. Dermatoendocrinol 2013;5:34-37.

6. Humayun Q, Iqbal R, Azam I, Khan AH, Siddiqui AR, Baig-Ansari N. Development and validation of sunlight exposure measurement (SEM-Q) for use in adult population residing in Pakistan. BMC Public Health 2012;12:421.

7. Wu S, Ho SC, Lam TP, Woo J, Yuen PY, Qin L, et al. Development and validation of a lifetime exposure questionnaire for use among Chinese populations. Sci Rep 2013;3:2793.

8. Hanwell HE, Vieth R, Cole DE, Scillitani A, Modoni S, Frusciante $\mathrm{V}$, et al. Sun exposure questionnaire predicts circulating 25-hydroxyvitamin D concentrations in Caucasian hospital workers in southern Italy. J Steroid Biochem Mol Bio 2010;121:334-337.

9. Cargill J, Lucas RM, Gies P, King K, Swaminathan A, Allen MW, et al. Validation of brief questionnaire measures of sun exposure and skin pigmentation against detailed and objective measures including vitamin D status. Photochem Photobiol 2013;89:219226.

10. Tabachnick BG, Fidell LS. Using multivariate statistics. 5th ed. Boston: Pearson Education; 2006, p. 633.

11. Yu MG, Castillo-Carandang N, Sison ME, Uy AB, Villarante KL, Maningat MP, et al. Attitudes, behaviors, and beliefs of urban adult Filipinos on sunlight exposure: a qualitative study. JAFES 2018;33:37-43.

12. Siniscalco MT, Auriat N. Quantitative research methods in educational planning: module 8 questionnaire design; 2005 [cited 2018 Nov 5]. Available from: http://unesdoc.unesco.org/images/ 0021/002145/214555E.pdf.

13. Lynn MR. Determination and quantification of content validity. Nurs Res 1985;35:382-385.

14. World Health Organization. Process of translation and adaptation of instruments [cited 2018 Nov 5]. Available from: http:// www.who.int/substance_abuse/research_tools/translation/en/.

15. Hair JF, Black WC, Babin BJ, Anderson RE, Tatham RL. Multivariate data analysis. 6th ed. New Jersey: Pearson Education; 2006, p. 123.

16. Latha MS, Martis J, Shobha V, Sham Shinde R, Bangera S, Krishnankutty B, et al. Sunscreening agents: a review. J Clin Aesthet Dermatol 2013;6:16-26.

17. Holick MF. The role of vitamin D for bone health and fracture 
prevention. Curr Osteoporos Rep 2006;4:96-102.

18. ISC-Audubon. The Köppen climate classification system [cited 2018 Nov 5]. Available from: http://www.thesustainabilitycouncil. org/resources/the-koppen-climate-classification-system/.

19. Torres A. Kinship and social relations in Filipino culture; 1985 [cited 2018 Nov 5]. Available from: http://lynchlibrary.pssc.org. ph:8081/ handle/0/1221.

20. Burns AC, Veeck A, Bush FF. Marketing research. 8th ed. Harlow; Pearson; 2017, p. 245.

21. Converse JM, Presser S. Survey questions: handcrafting the standardized questionnaire. Beverly Hills: Sage Publications; 1986, p.
35-36.

22. Oppenheim AN. Questionnaire design, interviewing and attitude measurement. London: Pinter Publishers; 1992, p. 195-200.

23. ETS TOEFL. Test and score data summary for TOEFL internetbased and paper tests; 2010 [cited 2018 Nov 5]. Available from: https://www.ets.org/Media/Research/pdf/TOEFL-SUM-2010. pdf.

24. Burns KE, Duffett M, Kho ME, Meade MO, Adhikari NK, Sinuff $\mathrm{T}$, et al. A guide for the design and conduct of self-administered surveys of clinicians. CMAJ 2008;179:245-252. 